\title{
IFU observations of the core of Abell 2218
}

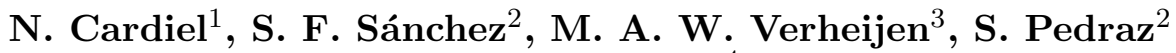 \\ and G. Covone ${ }^{4}$ \\ ${ }^{1}$ Departamento de Astrofísica, Universidad Complutense de Madrid, Spain \\ email: ncl@astrax.fis.ucm.es \\ ${ }^{2}$ Centro Astronómico Hispano Alemán, Calar Alto, Almería, Spain \\ ${ }^{3}$ Kapteyn Astronomical Institute, Groningen, The Netherlands \\ ${ }^{4}$ INAF-Osservatorio Astronomico di Capodimonte, Naples, Italy
}

\begin{abstract}
We present a study of the morphologies and stellar content of the galaxies in the central $\sim 74 " \times 64 "$ region of the galaxy cluster Abell 2218 (see Fig. 1). The spectroscopic data were obtained with the integral field unit PMAS (Roth et al. 2005) in the PPAK mode (Verheijen et al. 2004; Kelz et al. 2006), at the $3.5 \mathrm{~m}$ telescope of the Calar Alto Observatory. The covered wavelength range was $4650-8000 \AA$, with a spectral resolution FWHM $\sim 10 \AA$. These data were combined with with deep HST/ACS F475W, F555W, F625W and F850LP imaging, and additional data from the literature.

In Fig. 2 we show the rest-frame $B-V$ vs. $M_{V}$ colour-magnitude diagram for the identified cluster members. The data are segregated according to an objective morphological classification based on the Sérsic index. Contrary to previous results (e.g. Ziegler et al. 2001; Smail et al. 2001) there is a clear indication that the cluster population is not dominated by early-type galaxies, and that there exists an almost parity between early- and late-type objects. In addition, the late-type galaxies spread over a wider range of colours and luminosities with respect to the early-types. This indicates that early-type objects are more massive and have older stellar populations, while late-type galaxies are less massive and exhibit a wider range of stellar population properties.

The results agree with the two-steps scenario for the evolution of galaxies in clusters (e.g. Poggianti 2003), which explains the co-existence of a primordial population of early-type galaxies formed at early epochs, with an additional population of late-type galaxies that have been captured by the cluster, infalling, suffering a short enhancement of the star formation that is later quenched by the interaction with the environment, and that afterwards evolved passively, becoming redder and fainter. Finally, galaxy-galaxy interactions and dry merging processes were responsible for the building up of new massive spheroidal galaxies.
\end{abstract}

Full details are given in Sánchez et al. (2007).

Keywords. galaxies: clusters: general, galaxies: clusters: individual (Abell 2218), galaxies: elliptical and lenticular, cD, galaxies: spiral, galaxies: irregular, galaxies: stellar content, instrumentation: spectrographs

\section{References}

Kelz, A., Verheijen, M.A.W., Roth, M.M., et al. 2006, PASP, 118, 129

Poggianti, B.M. 2003, Ap\&SS, 285, 121

Roth, M.M., Kelz, A., Fechner, T., et al. 2005, PASP, 117, 620

Sánchez, S.F., Cardiel, N., Verheijen, M.A.W., Pedraz, S. \& Covone, G. 2007, MNRAS, in press (astro-ph/0611660)

Smail, I., Kuntschner, H., Kodama, T., et al. 2001, MNRAS, 323, 839

Verheijen, M.A.W., Bershady, M.A., Andersen, D.R., et al. 2004, Astronomische Nachrichten, 325,151

Ziegler, B.L., Bower, R.G., Smail, I., et al. 2001, MNRAS, 325, 1571 

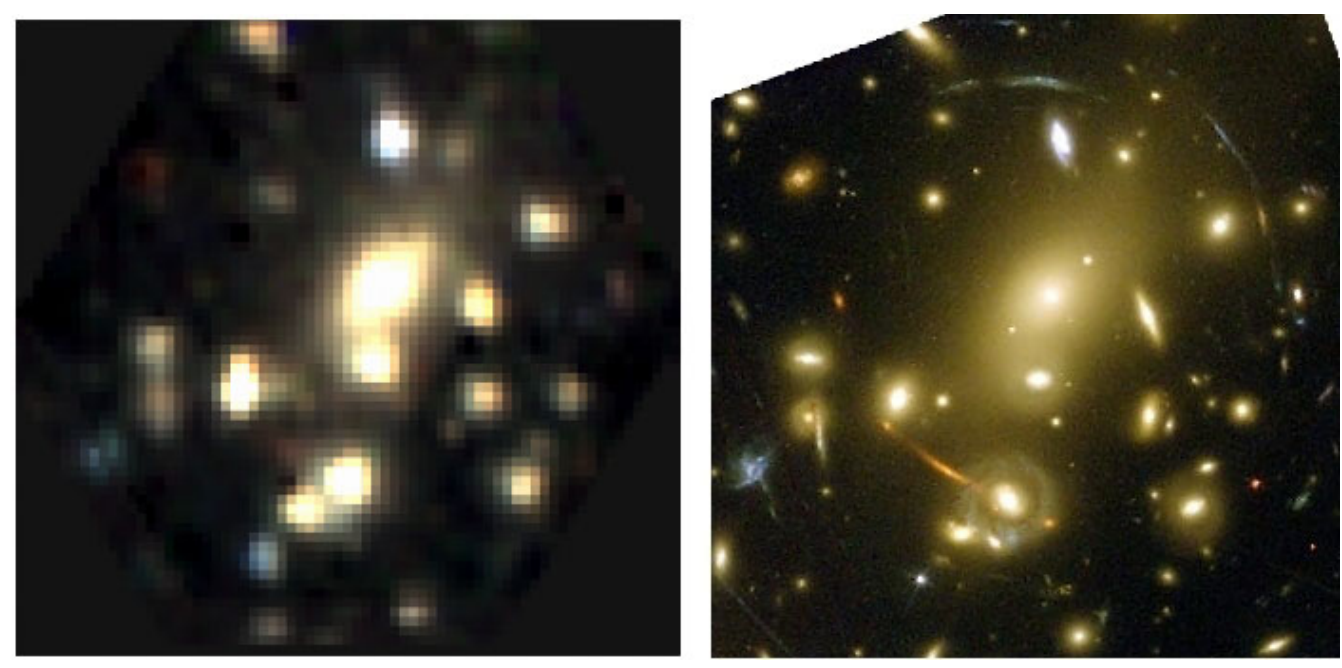

Figure 1. Left panel: Three-color image created by coadding the flux of the final IFU datacube through three broad-bands corresponding approximately to $B, R$ and $I$. Right panel: Similar image created from HST/ACS data.

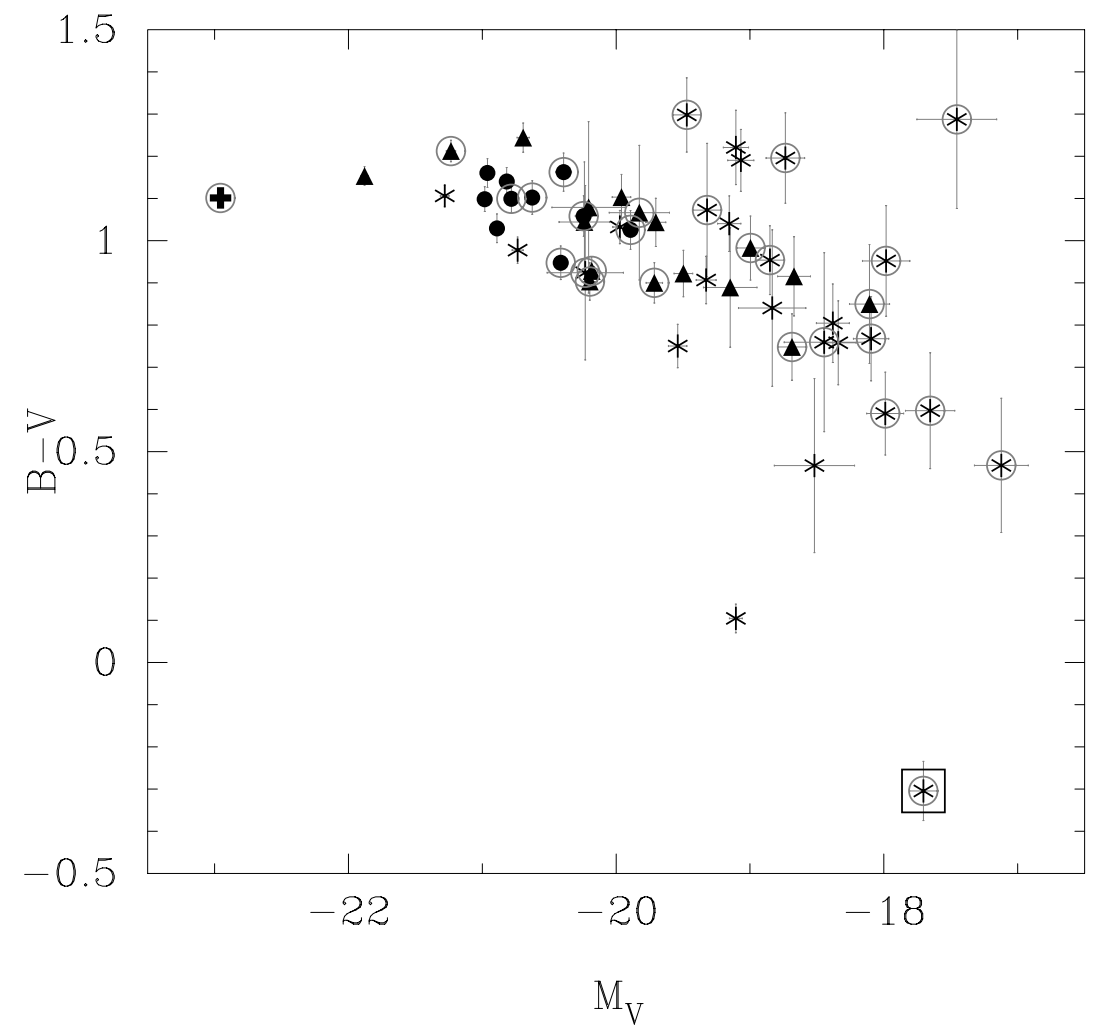

Figure 2. Rest-frame $B-V$ color distribution as a function of absolute magnitude $M_{V}$. Solid circles indicate early-type galaxies (Sérsic index $n>2.5$ ), asterisks are late-type galaxies $(n<1.75$ ), whereas filled triangles correspond to lenticular galaxies $(1.75 \leqslant n \leqslant 2.5)$. The $\mathrm{cD}$ galaxy is shown as a filled cross (top left), and the only galaxy with a high asymmetry index is surrounded by a big open square (bottom right). The 28 encircled galaxies belong to our sample, observed within the field-of-view of PMAS (in the PPAK mode). 